\title{
A von Hamos x-ray spectrometer based on a segmented-type diffraction crystal for single-shot $x$-ray emission spectroscopy and time-resolved resonant inelastic $x$-ray scattering studies
}

\author{
J. Szlachetko, ${ }^{1,2, a)}$ M. Nachtegaal,,${ }^{1, b)}$ E. de Boni, ${ }^{1}$ M. Willimann, ${ }^{1}$ O. Safonova, ${ }^{1}$ J. Sa, ${ }^{1}$ \\ G. Smolentsev, ${ }^{1}$ M. Szlachetko, ${ }^{1}$ J. A. van Bokhoven, ${ }^{1,3}$ J.-Cl. Dousse, ${ }^{4}$ J. Hoszowska, ${ }^{4}$ \\ Y. Kayser, ${ }^{4}$ P. Jagodzinski, ${ }^{5}$ A. Bergamaschi, ${ }^{1}$ B. Schmitt, ${ }^{1}$ C. David, ${ }^{1}$ and A. Lücke ${ }^{1}$ \\ ${ }^{1}$ Paul Scherrer Institut, 5232 Villigen PSI, Switzerland \\ ${ }^{2}$ Institute of Physics, Jan Kochanowski University, 25-406 Kielce, Poland \\ ${ }^{3}$ Institute for Chemical and Bioengineering, ETH Zurich, 8093 Zürich, Switzerland \\ ${ }^{4}$ Department of Physics, University of Fribourg, 1700 Fribourg, Switzerland \\ ${ }^{5}$ University of Technology, Kielce, Poland
}

(Received 9 July 2012; accepted 17 September 2012; published online 9 October 2012)

\begin{abstract}
We report on the design and performance of a wavelength-dispersive type spectrometer based on the von Hamos geometry. The spectrometer is equipped with a segmented-type crystal for x-ray diffraction and provides an energy resolution in the order of $0.25 \mathrm{eV}$ and $1 \mathrm{eV}$ over an energy range of $8000 \mathrm{eV}-9600 \mathrm{eV}$. The use of a segmented crystal results in a simple and straightforward crystal preparation that allows to preserve the spectrometer resolution and spectrometer efficiency. Application of the spectrometer for time-resolved resonant inelastic x-ray scattering and single-shot x-ray emission spectroscopy is demonstrated. (c) 2012 American Institute of Physics. [http://dx.doi.org/10.1063/1.4756691]
\end{abstract}

\section{INTRODUCTION}

High energy resolution hard $\mathrm{x}$-ray $(5-15 \mathrm{keV})$ emission spectroscopy (XES) is a powerful tool to determine the electronic structure of matter and has found many applications in chemistry, ${ }^{1}$ geology, ${ }^{2}$ nanotechnology, ${ }^{3}$ magnetism, ${ }^{4}$ catalysis, ${ }^{5}$ and biology, ${ }^{6}$ to name of few. At synchrotron sources, hard x-ray emission spectroscopy is used for resonant inelastic x-ray scattering studies (RIXS), ${ }^{7}$ high energy resolution fluorescence detected XAS spectroscopy (HERFDXAS or alternatively called partial fluorescence yield XAS) (Refs. 8-11) and to study core-to-core and valence-to-core transitions. ${ }^{12-14}$ The high energy resolution XAS/RIXS techniques require the experimental resolution to be lower than the natural lifetime broadening of the excited state. For $\mathrm{x}$-rays between $5 \mathrm{keV}$ and $15 \mathrm{keV}$, the lifetime broadening of the K-shell is in the range of $1 \mathrm{eV}-5 \mathrm{eV}$ (i.e., lifetimes of $1.6-6.5 \times 10^{-16} \mathrm{~s}$ ), while for the L-shell it is between $3 \mathrm{eV}$ and $10 \mathrm{eV}\left(0.7-2.1 \times 10^{-16} \mathrm{~s}\right) .{ }^{15}$ If the experimental resolution is below the $\mathrm{K}$ - or L-lifetime broadening, an enhanced sensitivity on the measured XAS/RIXS spectral shape can be obtained. ${ }^{8,9}$ High energy resolution XAS is used to follow the dynamic changes of the chemical state, electronic and geometric structure of matter under reaction conditions. ${ }^{16}$ In contrast, RIXS spectroscopy is usually performed under steadystate conditions, due to its relatively large acquisition time ranging from several minutes to a few hours.

Detection of the emission from core-to-core transitions is commonly used for XAS and RIXS spectroscopy. These inner-shell electron transitions provide high probability rates that are needed to measure weak RIXS signals. The core-

\footnotetext{
a)Electronic mail: jakub.szlachetko@psi.ch.

b) Electronic mail: maarten.nachtegaal@psi.ch.
}

electronic states are usually weakly interacting with the outermost electrons leading to a simple description of the initial and final electronic configurations. In case of core-to-core XAS and RIXS measurements, the multiple splitting of the final state can generally be neglected, making the comparison with theoretical XAS simulations straightforward and direct. ${ }^{11}$ On the other hand, core-to-core XES can be used as a probe of inner shell electron correlations, multi-electron scattering and electron rearrangement processes. ${ }^{17}$

High energy resolution XES enables to probe the ligand environment of a metal of interest. ${ }^{12,18-22}$ Access to the ligand orbital is obtained by ionizing the core electron and measuring the following valence-to-core transition. This decay channel involves the valence electrons of the ligands and therefore provides information about the highest occupied electronic levels in matter. Thanks to the penetrating properties of hard $\mathrm{x}$-rays, valence-to-core spectroscopy allows to study bulk materials under in situ conditions which is a limiting factor for electron based techniques. Unlike XAS and RIXS, valenceto-core measurements do not require a monochromatic and tunable x-ray source and therefore can be applied for chemical investigations using laboratory sources. ${ }^{23,24}$ Furthermore, no need for the beam monochromacity makes valence-to-core spectroscopy a well suitable technique for $\mathrm{x}$-ray free electron laser sources (XFEL). The XFEL source provides a very intense femtosecond $\mathrm{X}$-ray pulse, which has a large energy bandwidth $(\Delta \mathrm{E} / \mathrm{E} \sim 0.2 \%)$ and a large variation of the shotto-shot mean energy. To fully explore these short and intense pulses for electronic structure determination through valenceto-core transitions, single-shot capability instruments are of prime importance.

In general, high energy resolution XES relies on the $\mathrm{x}$-ray dispersion by a crystal and is governed by the Bragg law. Wavelength-dispersive spectrometers are usually based 
on a curved crystal geometry. ${ }^{25-28}$ One- or two-dimensional bending of the crystal results in improvement of the spectrometer efficiency, but also introduces a loss in energy resolution due to the deformation and waviness of the curved crystal. ${ }^{29}$ For this reason, the bending radius of the crystal has to be kept relatively large to maintain sub eV spectral resolution. ${ }^{30-33}$ To avoid elastic deformations caused by bending, diced analyzer crystals were developed for the Johanntype spectrometers, ${ }^{34-36}$ providing an experimental resolution in the meV range. A similar design, based on multiple crystals fixed in one 2D bent substrate was proposed for microprobe XES spectrometers. It was demonstrated that based on a multiple-crystal array affixed on a spherical substrate, a good energy resolution and a large solid collection angle can be achieved. ${ }^{37-39}$ The use of diced or segmented-type optics leads to a simple spectrometer that is easy to implement for any scattering geometry.

We report on a wavelength-dispersive spectrometer based on a segmented-type crystal and a 1D array detector arranged in the von Hamos geometry. The spectrometer geometry allows recording a wide energy range $\mathrm{x}$-ray emission spectrum at a single excitation energy and has found a variety of applications in combination with lab, synchrotron, and XFEL sources. ${ }^{30,40-44}$ Thanks to the dispersive-type of diffraction, the spectrometer geometry provides the possibility of a single-shot measurement with a total energy bandwidth ranging from a few tens to a few hundreds of $\mathrm{eV}$. The development of innovative and compact von Hamos spectrometers has also benefited from the parallel advances of position-sensitive detectors. In the present paper a continuously bent crystal, typically used in the von Hamos geometry, is replaced by a segmented crystal. The spectrometer, operated at a $25 \mathrm{~cm}$ focusing radius, was equipped with a $\mathrm{Si}(111)$ crystal consisting of 20 segments each measuring $5 \times 50 \mathrm{~mm}^{2}$ (focusing $\times$ dispersing). The diffracted $\mathrm{x}$-rays were recorded by a single photon counting strip-type detector, which was originally developed for time-resolved XRD. ${ }^{45}$ We present the design, crystal preparation procedure, and the performance achieved with the spectrometer. The application of the spectrometer for single-shot XES and quick-RIXS measurements is demonstrated.

\section{SPECTROMETER GEOMETRY AND EXPERIMENTAL SETUP}

The von Hamos geometry is schematically represented in Fig. 1(a). In this arrangement, the x-ray fluorescence from the sample is diffracted by the cylindrically bent crystal and recorded by a position sensitive detector. On the detector plane, the diffracted $\mathrm{x}$-rays create a $2 \mathrm{D}$ image. On the axis of dispersion, the position of detected x-rays is directly correlated to the energy/wavelength (Fig. 1(b)). The energy range of the detected $\mathrm{x}$-rays is determined primarily by the length of the crystal and the detector along the axis of dispersion. The one-dimensional bending of the crystal enhances the efficiency of the instrument by providing focusing in the nondispersion plane. For crystals with an ideally bent surface, the focal spot size on the detector will match the $\mathrm{x}$-ray source size (Fig. 1(c)). To change the Bragg angles domain, a linear
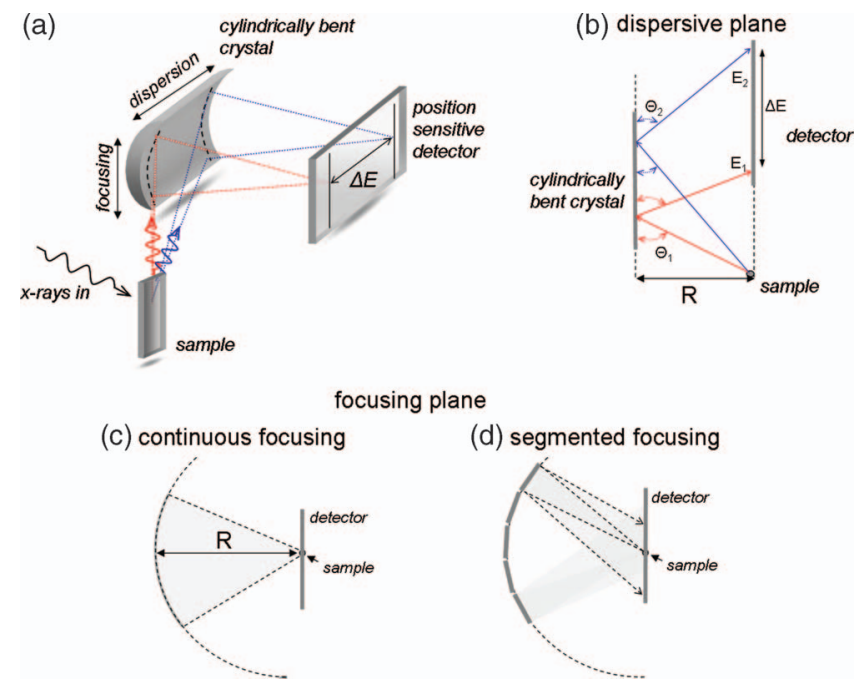

FIG. 1. (a) Schematic of the von Hamos geometry. (b) Projection along the axis of dispersion, (c) projection along focusing plane, (d) the same as (c) but for segmented-type of focusing.

displacement of the crystal and detector along the axis of dispersion is needed. The position of the crystal center is given by $\mathrm{R} \times \cot (\theta)$, where $\mathrm{R}$ is the radius of curvature and $\theta$ the Bragg angle. The detector distance from the sample is twice that of the crystal.

The main factors influencing the energy resolution and the efficiency of the spectrometer are the bending radius of the crystal, the source size, and the spatial resolution of the detector in the dispersion plane. Those factors are correlated and should be carefully considered for any spectrometer design. For an optimal performance, the spatial resolution of the detector should be matched to the source size on the sample. The bending of the crystal provides focusing of the $\mathrm{x}$-rays and the radius of curvature defines the source-to-crystal and crystal-to-detector distances. However, one-dimensional bending in the non-dispersion axis also influences the dispersion plane of the crystal, due to the waviness and distortions in both, focusing and dispersion directions. A relatively large bending radius ensures high energy resolution but results in lower efficiency due to the decrease of solid angle. On the other hand, increased efficiency is achieved at the cost of energy resolution at smaller bending radii.

The application of a segmented-type crystal for focusing permits to preserve the intrinsic energy resolution of a flat crystal. As shown schematically in Fig. 1(d), in such a geometry, the crystal consists of segments which are tangent in the focusing plane to the circle defined by the radius of curvature. This arrangement results in quasi-focusing of the $\mathrm{x}$-rays and in an enlarged $x$-ray footprint on the detector. However, in the dispersion plane, $\mathrm{x}$-ray diffraction always takes place on the flat-crystal segment.

The total solid angle $(\Omega)$ covered by a segmented-type crystal can be approximated by the ratio of the crystal area perpendicular to the incident radiation to the squared distance between the crystal and the source (see Fig. 2(a)):

$$
\Omega=N_{s} \cdot \frac{S_{w} \cdot S_{h}}{R^{2}} \cdot \sin ^{3}(\theta)
$$


(a)

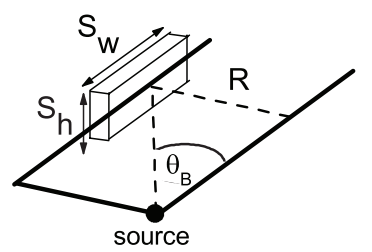

(b)

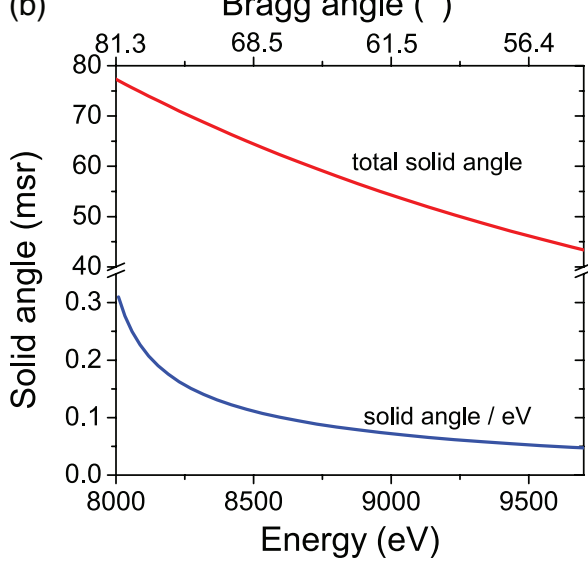

FIG. 2. (a) Schematic representation of von Hamos geometry for solid angle calculations, (b) the energy dependence of total solid angle (red line), and solid angle per $\mathrm{eV}$ for $25 \mathrm{~cm}$ radius of curvature.

where $N_{s}$ is the number of crystal segments, and $S_{w}$ and $S_{h}$ the segment width and segment height, respectively. R stands for radius of crystal curvature and $\theta$ is the Bragg angle. As an example, the total solid angle is plotted in Fig. 2(b) (red line), assuming a $\mathrm{Si}(444)$ crystal consisting of 20 segments, with a segment size of $5 \mathrm{~mm}$ high and $50 \mathrm{~mm}$ wide. The radius of curvature was set to $25 \mathrm{~cm}$. The total solid angle is in the range of 44-76 msr (milisteradian) for Bragg angles between $55^{\circ}$ and $80^{\circ}$. Using Eq. (1), the solid angle per unit energy $\left(\Omega_{\mathrm{eV}}\right)$ can be calculated by replacing the segment width with the length of the crystal contributing to the x-ray diffraction. The following formula is obtained for $\Omega_{\mathrm{eV}}$ :

$$
\Omega_{\mathrm{eV}}=\frac{N_{s} \cdot S_{h}}{R} \times \frac{\sin ^{2}(\theta)}{\cos (\theta) \cdot E},
$$

where $E$ stand for the energy in $\mathrm{eV}$ of the diffracted x-rays. From Eq. (2), one sees that the solid angle per eV is proportional to the total height of the crystal and inversely proportional to the radius of curvature. The variation of $\Omega_{\mathrm{eV}}$ in the x-ray energy range of interest is plotted in Fig. 2 (blue line). For the above-mentioned Bragg angles of $55^{\circ}$ and $80^{\circ}$, values of about $50 \times 10^{-3}$ and $280 \times 10^{-3} \mathrm{msr} / \mathrm{eV}$, respectively, are found.

The absolute efficiency of the spectrometer per $\mathrm{eV}$ can be calculated from the following formula:

$$
E f f_{\mathrm{eV}}=\left(\Omega_{\mathrm{eV}} / 4 \pi\right) \cdot R_{C r y} \cdot D_{E f f},
$$

where $R_{C r y}$ is the crystal peak reflectivity and $D_{E f f}$ stands for the detector efficiency. For x-ray energies between $8000 \mathrm{eV}$ and $9600 \mathrm{eV}$, the $\mathrm{Si}(444)$ crystal peak reflectivity is in the range of $90 \%$ (Ref. 46) while the detector efficiency is at the level of $85 \%-90 \% .{ }^{47}$ According to Eq. (3), this leads to a spectrometer efficiency varying from about $21 \times 10^{-4}$ at $8000 \mathrm{eV}$ down to $3 \times 10^{-4}$ at $9600 \mathrm{eV}$. However, as recently demonstrated, the spectrometer efficiency can be further enhanced, by increasing the solid angle by a multi-crystal arrangement. ${ }^{44}$

Assuming Gaussian distributions for the different contributions to the spectrometer energy resolution $(\Delta E)$, the latter can be written

$$
\Delta E=\sqrt{\Delta E_{B}^{2}+\Delta E_{s}^{2}+\Delta E_{S e g m}^{2}+\Delta E_{D e t}^{2}} .
$$

In (4), $\Delta E_{B}$ which is equal to $E \cdot \Delta \theta \cdot \operatorname{Cot}(\theta)$ accounts for the Darwin width $\Delta \theta$ of the crystal and $\Delta E_{s}$ for the source size. The effect of crystal segmentation is expressed by $\Delta E_{\text {Segm }}$ and depends on the crystal segment size as well as on the crystal radius of curvature. For $\mathrm{R} \rightarrow$ inf the $\Delta E_{\text {Segm }}$ contribution approaches 0 . The last factor $\Delta E_{D e t}$ includes the spatial resolution of the position sensitive detector. Assuming an x-ray energy of $8000 \mathrm{eV}$, a $\mathrm{Si}(444)$ diffraction, a $100 \mu \mathrm{m}^{2}$ source size and $50 \mu \mathrm{m}$ spatial resolution of the detector, the following $\Delta E$ contributions are obtained: $\Delta E_{B}=0.05 \mathrm{eV}$, $\Delta E_{s}=0.2 \mathrm{eV}$, and $\Delta E_{D e t}=0.1 \mathrm{eV}$. For the $\Delta E_{\text {Segm }}$ factor, we used a segment size of $5 \mathrm{~mm}$ in the direction of dispersion and a radius of curvature of $25 \mathrm{~cm}$. The estimated $\Delta E_{\text {Segm }}$ contribution was found to be at the level of $0.1-0.15 \mathrm{eV}$. Based on the above estimations, a total spectrometer resolution of about $0.3 \mathrm{eV}$ was calculated. We would like to point out that all the factors contribute almost equally $(0.1-0.2 \mathrm{eV})$ to the spectrometer resolution indicating thus an optimal spectrometer performance in terms of energy resolution. For much shorter radii of curvatures (e.g., $\mathrm{R}=10 \mathrm{~cm}$ ) both the contribution of the source size and crystal segmentation would be increased by $1-2 \mathrm{eV}$, leading to a significant deterioration of the spectrometer resolution.

The von Hamos spectrometer was designed for a radius of curvature of $25 \mathrm{~cm}$ which is a compromise between several constraints. First, such a radius provides the required energy resolution and high efficiency of the spectrometer in combination with the beam size on the sample and spatial resolution of the x-ray detector. Second, it ensures enough space in the sample environment that allows the use of high-temperature and high-pressure cells. For x-ray detection, a MythenII striptype single-counting photon detector is employed. ${ }^{47}$ The detector area consists of 1280 strips of $50 \mu \mathrm{m}$ width and $8 \mathrm{~mm}$ height. The background events and low energy noise induced in the detector can be diminished by a low-energy discriminator set at a value of $5 \mathrm{keV}$. To access a wide range of Bragg angles, both the crystal and detector are mounted on parallel translation stages. The translation was defined to be $200 \mathrm{~mm}$ for the crystal and $400 \mathrm{~mm}$ for the detector providing the Bragg diffraction angles in the range between $90^{\circ}$ and $50^{\circ}$.

The spectrometer was installed at the SuperXAS beamline of the Swiss Light Source, Villigen, Switzerland. For the measurements, $x$-rays in the range from $8 \mathrm{keV}$ to $10 \mathrm{keV}$ were delivered by the $2.9 \mathrm{~T}$ super-bend magnet. A collimated beam was formed by means of a spherical Rh-coated mirror and monochromatized by double $\mathrm{Si}(111)$ crystals. Downstream of the monochromator, a Rh-coated torroidal mirror was employed to focus the incident $\mathrm{x}$-rays with a spot size of 


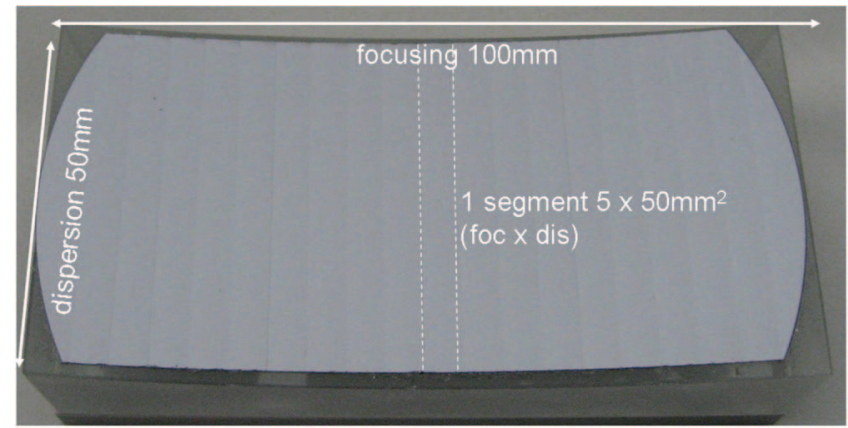

FIG. 3. Photograph of a segmented $\mathrm{Si}(111)$ crystal glued on a curved glass support.

$100 \times 100 \mu \mathrm{m}^{2}$ on the sample. The typical photon flux obtained with this configuration was about 3-4 $\times 10^{11}$ photons/s.

\section{CRYSTAL PREPARATION}

A segmented crystal for the von Hamos spectrometer was prepared from a $\mathrm{Si}(111)$ wafer measuring $10 \times 5 \mathrm{~cm}^{2}$. The crystal preparation consisted of two steps. First, the back side of the crystal was mechanically grooved by means of a diamond-saw at $5 \mathrm{~mm}$ equidistant steps in the focusing direction and $50 \mathrm{~mm}$ in the dispersing direction. The groove depth was slightly smaller than the thickness of the crystal $(300 \mu \mathrm{m})$. Second, the crystal was glued with a thin adhesive tape on a BK7-type cylindrical glass support from Eksma Optics having a radius of curvature of $25 \mathrm{~cm}$. In order to obtain uniform adhesion of the crystal to the support, a concave counterpart glass block was made and used to press the crystal onto the adhesive tape. As a result of the crystal grooving and the pressure exerted by the convex glass block, a wellcontrolled breaking of the crystal along the grooves could be achieved. As shown in Fig. 3, the procedure allows fabricating a segmented-crystal with low dead-volume caused by broken edges of the crystal.

\section{SPECTROMETER RESOLUTION}

To probe the performance of the spectrometer, a series of measurements of elastic scattering at several beam energies in the range between $8040 \mathrm{eV}$ and $9600 \mathrm{eV}$ were performed. An example of experimental spectrum recorded at an incidence beam energy of $8800 \mathrm{eV}$ is shown in Fig. 4(a). From the experiment we found that the spectrometer response can be well reproduced by a Gaussian function over the measured energy range.

As described in Eq. (4), the experimental resolution depends on the beam size on the sample, incident beam energy resolution, and the geometrical effects induced by the crystal segmentation. To distinguish these effects and determine influence on the spectrometer resolution, $\mathrm{x}$-ray tracing simulations were performed. The computations included the geometrical constraints of the von Hamos setup, the effects of crystal segmentation as well as the properties (size and energy resolution) of the incident $\mathrm{x}$-ray radiation. The $\mathrm{x}$-ray events were randomly generated for a $100 \mu \mathrm{m}^{2}$ source size assum-
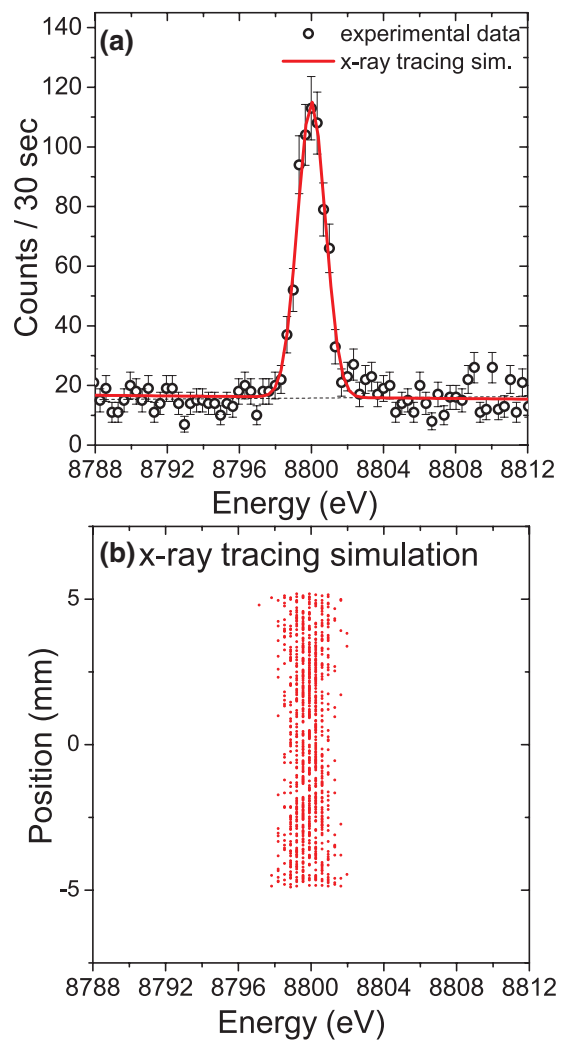

FIG. 4. (a) The elastic scattering peak (open circles) measured with the $\mathrm{Si}(111)$ diced crystal at a beam energy of $8800 \mathrm{eV}$. The red solid line corresponds to the results of $\mathrm{x}$-ray tracing simulations and (b) the calculated 2D distribution of the x-ray hit points on the detector plane. The projection of this distribution onto the energy axis is given by the red curve in the top panel.

ing a Gaussian distribution of the events density. The random $\mathrm{x}$-ray directions were chosen with a margin of $20 \%$ larger than the solid angle covered by the crystal. The x-ray paths from the source were computed for each event and the x-ray vector positions at the impact points on the crystal were calculated. The x-ray diffraction probability was considered using the shape of the crystal rocking curve taken from the XOP software. ${ }^{46}$ The vectors of the diffracted events were then projected onto the detector plane and the vertical and horizontal positions registered (see Fig. 3(b)). Typically $10^{7}$ events per simulated spectrum were generated.

As an example, the calculated distribution of the $\mathrm{x}$-ray hit points on the detector plane is drawn in Fig. 4(b) for an incident beam energy of $8800 \mathrm{eV}$. Each red dot in the graph corresponds to one "detected" event. To obtain a 1D x-ray spectrum, this x-ray image is projected onto the axis of dispersion. The resulting curve is plotted as a red solid line in Fig. 4(a) together with experimental data points. A good agreement between the measured and calculated spectra is obtained.

The fitted total experimental resolution versus photon energies is shown in Fig. 5. At the largest Bragg angle of $80^{\circ}$, which corresponds to an energy of $8040 \mathrm{eV}$, an experimental resolution (full width at half-maximum) of $1.25 \mathrm{eV}$ was obtained. The resolution degrades continously to $2.2 \mathrm{eV}$ when increasing the incident photon energy to $9600 \mathrm{eV}$.

Based on the simulations, we calculated the main contributions affecting the experimental resolution (Fig. 5). The 


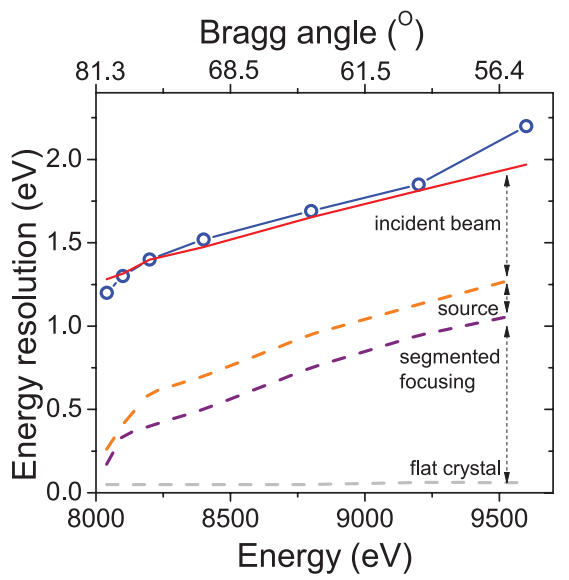

FIG. 5. The experimental energy resolution versus incident photon energy (blue) compared to the results of $\mathrm{x}$-ray tracing simulations (red). The calculated contributions to the total resolution are also shown: intrinsic crystal resolution (grey), effects of segmented focusing (purple), source size (orange), and energy resolution of incident beam.

intrinsic resolution of the flat crystal contributes at the level of $0.05 \mathrm{eV}-0.1 \mathrm{eV}$ (gray dashed line), while the contribution of the crystal segmentation varies between $0.2 \mathrm{eV}$ at $8040 \mathrm{eV}$ and about $1.0 \mathrm{eV}$ at $9600 \mathrm{eV}$. These two broadening effects determine the intrinsic energy resolution of the von Hamos spectrometer. A further decrease in resolution of about $10 \%$ is caused by the $100 \mu \mathrm{m}$ vertical source size. Including the broadening effect of the source size, a total energy resolution of $0.25 \mathrm{eV}$ at $8000 \mathrm{eV}$ is obtained, which is close to the value of $0.3 \mathrm{eV}$ deduced from Eq. (4). This indicates that the strain and figure errors resulting from the crystal preparation do not contribute in a significant way to the spectrometer resolution, probably because the crystal flatness in the direction of dispersion is well preserved and the pseudo-cylindrical shape of the crystal is well reproduced by the proper orientation of the segments. On the other side, no significant strain effect is observed that might be induced by breaking the crystal along the grooves. Finally, to obtain the total experimental resolution, the contribution of the incident beam energy distribution is included assuming a relative resolving power of $1.37 \times 10^{-4}$ for the double $\mathrm{Si}(111)$ monochromator.

\section{APPLICATION TO TIME-RESOLVED RIXS AND SINGLE-SHOT XES}

The dispersive-type of detection provided by the von Hamos geometry can be exploited to record time-resolved XES. Here, we show an example of time-resolved RIXS. In contrast to other focusing-type spectrometers for which an $\mathrm{X}$ ray emission line should be scanned point by point, in the von Hamos geometry the whole line can be recorded in oneshot allowing for very quick measurements of the full RIXS plane. This is an attractive feature, which may be applied in many applications for in situ spectroscopic studies of dynamic systems.

As an example, the full RIXS planes of $\mathrm{CuO}$ and $\mathrm{Cu}_{2} \mathrm{O}$ powder pellet samples were measured around the $\mathrm{K}$-absorption edge of $\mathrm{Cu}$ by detecting the $\mathrm{K} \alpha_{1,2}$ doublet fluorescence signal. The exposure time per one incident energy point was $0.2 \mathrm{~s}$ and the total acquisition time for each RIXS map was 7 s. The resulting RIXS planes are plotted in Fig. 6. The data quality allows to determine the chemical speciation of the element of interest. The non-resonant XES is extracted from the measurement taken at the highest incident energy (blue line). The high energy resolution XAS spectrum, which
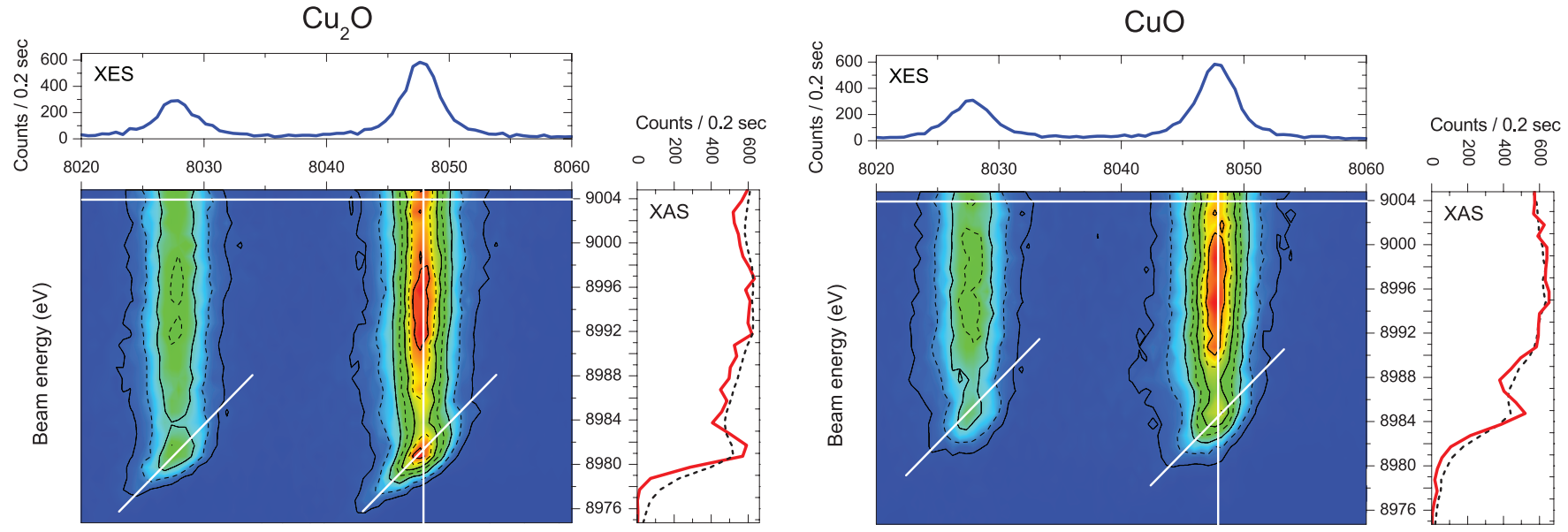

Emission energy (eV)
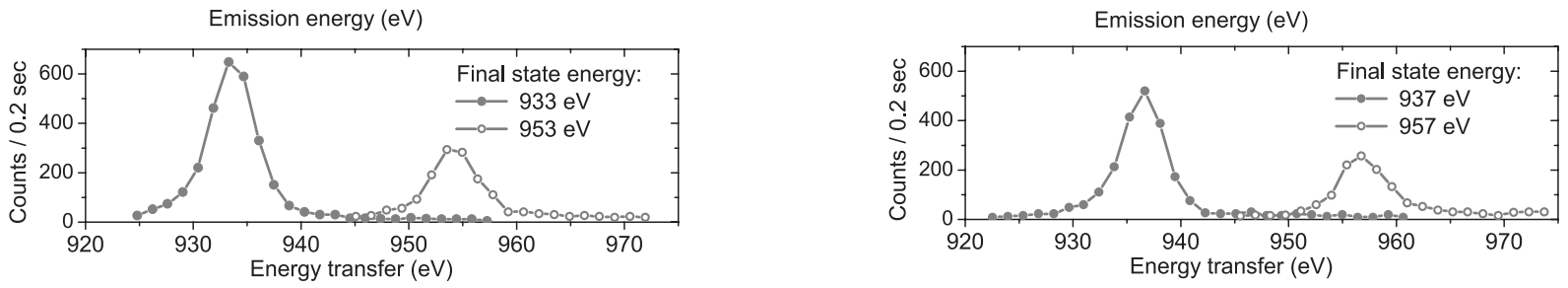

FIG. 6. The RIXS planes for $\mathrm{Cu}_{2} \mathrm{O}$ and $\mathrm{CuO}$ compounds recorded each in an acquisition time of only $7 \mathrm{~s}$. The blue lines (top panels) correspond to the nonresonant XES spectra and the red lines to the high energy resolution XAS spectra. The profiles obtained for a constant energy transfer are shown in the bottom panel (gray lines). 
is highly sensitive to the chemical environment, was extracted by integrating the intensity at the top of the $\mathrm{K} \alpha_{1}$ line (red line). For comparison, a conventional XAS recorded by means of total fluorescence yield is also plotted (black dashed line). As shown the high energy resolution XAS shows more detailed features around the absorption edge. In these measurements, the experimental resolution is given by the convolution of the spectrometer resolution at the $\mathrm{Cu} \mathrm{K} \alpha$ line $(8040 \mathrm{eV})$ and the incoming beam energy distribution around $8980 \mathrm{eV}$. At these energies, the spectrometer resolution amounts to $0.3 \mathrm{eV}$ while the incoming beam energy resolution is $1.23 \mathrm{eV}$. Thus, a total experimental resolution of $1.27 \mathrm{eV}$ is expected which is lower than the $\mathrm{Cu} \mathrm{K}$-shell broadening of $1.49 \mathrm{eV} .^{15}$ Spectra of similar quality were recorded using a spherical Johann-type spectrometer. ${ }^{48}$ The measurement of the RIXS plane also allows to extract the energy transfer curves at the strongest resonances. For illustration, the energy transfer plots of $\mathrm{Cu}_{2} \mathrm{O}$ (respectively, $\mathrm{CuO}$ ) corresponding to the final state energies of $933 \mathrm{eV}$ and $953 \mathrm{eV}$ (937 eV and $957 \mathrm{eV}$ ) and to a beam energy of $8981 \mathrm{eV}(8984 \mathrm{eV})$ for which a resonance is observed in the XAS spectrum are shown in the bottom panels of Fig. 6 .

To test the capability of the spectrometer to perform single-shot $\mathrm{K} \beta$ and valence-to-core spectroscopy at XFELs, XES spectra of a $\mathrm{CuO}$ powder pellet sample were recorded at a fixed excitation energy. The x-ray beam energy was tuned above the $\mathrm{K}$ edge of $\mathrm{Cu}$ to a value of $9050 \mathrm{eV}$. Two sets of spectra were measured. To determine the profile of the valence-to-core transition of $\mathrm{CuO}$, the $\mathrm{x}$-ray spectrum was recorded for long acquisition time resulting in a dose of $4.5 \times 10^{15}$ photons (Fig. 7 top). The measured spectrum consists of a strong $\mathrm{K} \beta_{1,3}$ emission line (at $8905 \mathrm{eV}$ ) accompanied by a weak valence-to-core transition located at around $8970 \mathrm{eV}$. As shown in the inset of Fig. 7, the valence-tocore spectrum consists of two main peaks located at $8975 \mathrm{eV}$ and $8960 \mathrm{eV}$, respectively. Assuming the $\mathrm{Cu} 1 s$ binding energy to be $8979 \mathrm{eV}$, the measured structures correspond to a $p$-projected highest occupied states with mean energies of $-4 \mathrm{eV}$ and $-19 \mathrm{eV} .^{49}$ This result is in agreement with previous $\mathrm{X}$-ray emission and XPS data. ${ }^{49,50} \mathrm{~A}$ second spectrum that was recorded at a total dose of $6 \times 10^{12}$ photons is plotted in the bottom panel of Fig. 7. The latter photon dose corresponds to the number of photons that are expected to be delivered by a few XFEL femtosecond pulses. As shown, the measured $\mathrm{K} \beta_{1,3}$ is of such quality that allows for a detailed analysis of the line shape and position. The main feature of the valence-to-core transition can also be observed. The fit of the straggling data evidences indeed a structure at $8975 \mathrm{eV}$, whereas the second peak at $8960 \mathrm{eV}$ could not be detected. Nevertheless, the present experiment proves the feasibility of single-shot $\mathrm{K} \beta$ measurements for real-time chemical speciation at fourth generation x-ray sources. For valence-to-core spectroscopy the XFEL experiments will be very challenging and difficult at single-shot due to the low intensities of these transitions. Actually, single-shot valence-to-core measurements are probably feasible only for solid or concentrated samples. In addition, in this case, sample refreshment frequencies similar to the $\mathrm{x}$-ray pulse repetition rates and a multi-crystal arrangement would probably be mandatory to

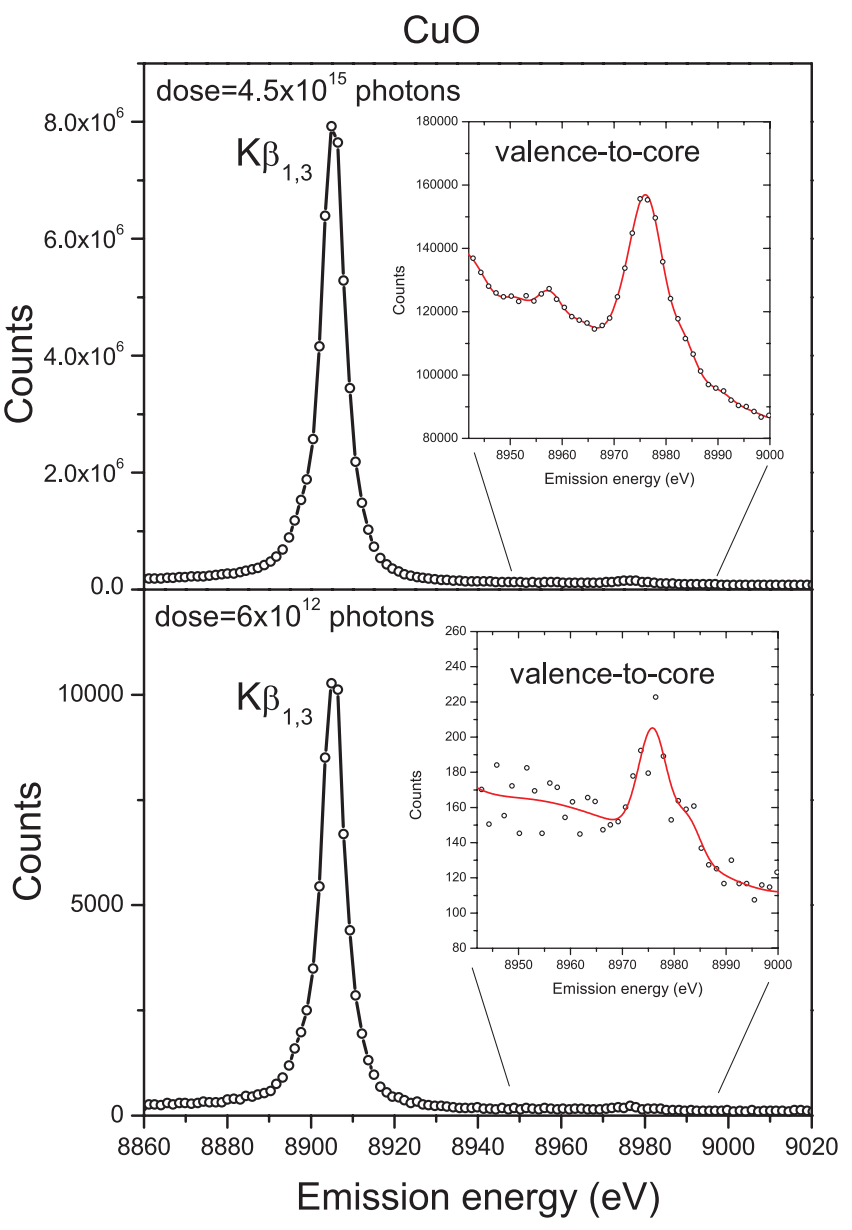

FIG. 7. $\mathrm{K} \beta_{1,3}$ and valence-to-core $\mathrm{x}$-ray emission for $\mathrm{CuO}$ recorded without scanning any beamline nor spectrometer component during acquisition. (Top) The acquisition times were chosen to correspond to absorption doses of $4.5 \times 10^{15}$ photons (top panel) and $6 \times 10^{12}$ photons (bottom panel), respectively.

perform successful single-shot measurements. In case of diluted, gaseous or liquid samples, the accumulation of several spectra/pulses would be necessary to obtain valence-to-core spectra of decent quality.

\section{SUMMARY}

We report on the development of the von Hamos spectrometer equipped with a segmented-type diffraction crystal. The intrinsic energy resolution of the instrument was found to be below eV level over the complete $\mathrm{x}$-ray energy range. Thanks to the dispersive-type of detection provided by the von Hamos geometry, in combination with a single photon counting micro-strip detector, the 2D RIXS planes were recorded at the acquisition time of a few seconds for different $\mathrm{Cu}$ oxides. The capability of the spectrometer to perform single-shot $\mathrm{K} \beta$ XES measurements is discussed and the feasibility study on application to XFEL sources is presented.

${ }^{1}$ G. Vanko, P. Glatzel, V.-T. Pham, R. Abela, D. Grolimund, C. N. Borca, S. L. Johnson, C. J. Milne, and C. Bressler, Angew. Chem., Int. Ed. 49, 5910 (2010).

${ }^{2}$ J. Badro, G. Fiquet, F. Guyot, J.-P. Rueff, V. V. Struzhkin, G. Vanko, and G. Monaco, Science 300, 789 (2003). 
${ }^{3}$ J. Szlachetko, D. Banas, A. Kubala-Kukus, M. Pajek, W. Cao, J.-Cl. Dousse, J. Hoszowska, Y. Kayser, M. Szlachetko, M. Kavcic, M. Salome, and J. Susini, J. Appl. Phys. 105, 086101 (2009).

${ }^{4}$ M. Sikora, A. Juhin, T.-C. Weng, P. Sainctavit, C. Detlefs, F. de Groot, and P. Glatzel, Phys. Rev. Lett. 105, 037202 (2010).

${ }^{5}$ P. Glatzel, J. Singh, K. O. Kavashnina, and J. A. van Bokhoven, J. Am. Chem. 132, 2555 (2010).

${ }^{6}$ K. M. Lancaster, M. Roemelt, P. Ettenhuber, Y. Hu, M. W. Ribbe, F. Neese, U. Bergmann, and S. DeBeer, Science 334, 974 (2011).

${ }^{7}$ A. Kotani and S. Shin, Rev. Mod. Phys. 73, 203 (2001).

${ }^{8}$ P. Eisenberger, P. M. Platzman, and H. Winick, Phys. Rev. Lett. 36, 623 (1976).

${ }^{9}$ K. Hämäläinen, D. P. Siddons, J. B. Hastings, and L. E. Berman, Phys. Rev. Lett. 67, 2850 (1991).

${ }^{10}$ U. Bergmann, P. Glatzel, J. H. Robblee, J. Messinger, C. Fernandez, R. Cinco, H. Visser, K. McFarlane, E. Bellacchio, S. Pizarro, K. Sauer, V. K. Yachandra, M. P. Klein, B. L. Cox, K. H. Nealson, and S. P. Cramer, J. Synchrotron Radiat. 8, 199 (2001).

${ }^{11}$ O. V. Safonova, M. Tromp, J. A. van Bokhoven, J. Evans, F. M. F. de Groot, and P. Glatzel, J. Phys. Chem. B 110, 16162 (2006).

${ }^{12}$ V. V. Nemoshkalenko and M. A. Mindlina, Phys. Status Solidi 25, K83 (1968).

${ }^{13}$ G. Doring, C. Sternemann, A. Kaprolat, A. Mattila, K. Hamalainen, and W. Schulke, Phys. Rev. B 70, 085115 (2004).

${ }^{14}$ V. A. Safonov, L. N. Vykhodtseva, Y. M. Polukarov, O. V. Safonova, G. Smolentsev, M. Sikora, S. G. Eeckhout, and P. Glatzel, J. Phys. Chem. B 110, 23192 (2006)

${ }^{15}$ J. L. Campbell, At. Data Nucl. Data Tables 77, 50 (2001).

${ }^{16}$ J. A. van Bokhoven, Phys. Chem. Chem. Phys. 12, 5502 (2010).

${ }^{17}$ J. Hoszowska, A. K. Kheifets, J.-Cl. Dousse, M. Berset, I. Bray, W. Cao, K. Fennane, Y. Kayser, M. Kavcic, J. Szlachetko, and M. Szlachetko, Phys. Rev. Lett. 102, 073006 (2009).

${ }^{18}$ T. B. Shashkina, Phys. Status Solidi 44, 571 (1971).

${ }^{19}$ S. A. Nemnonov and V. A. Trofimova, Phys. Status Solidi 55, K111 (1973).

${ }^{20}$ U. Bergmann, C. Horne, T. Collins, J. Workman, and S. Cramer, Chem. Phys. Lett. 302, 119 (1999).

${ }^{21}$ O. V. Safonova, M. Florea, J. Bilde, P. Delichere, and J. M. M. Millet, J. Catal. 268, 156 (2009).

${ }^{22}$ C. J. Pollock and S. DeBeer, J. Am. Chem. Soc. 133, 5594 (2011).

${ }^{23}$ M. Kavcic, J.-Cl. Dousse, J. Szlachetko, and W. Cao, Nucl. Instrum. Methods Phys. Res. B 260, 642 (2007).

${ }^{24}$ S. Fazinic, L. Mandic, M. Kavcic, and I. Bozicevica, Spectrochim. Acta, Part B 66, 461 (2011).

${ }^{25}$ H. H. Johann, Z. Phys. 69, 185 (1931).

${ }^{26}$ L. von Hamos, Naturwiss. 20, 705 (1932).

${ }^{27}$ T. Johannson, Z. Phys. 71, 507 (1933).

${ }^{28}$ J. W. M. DuMond, Rev. Sci. Instrum. 18, 626 (1947).

${ }^{29}$ S. Tagaki, Acta Crystallogr. 15, 1311 (1933).

${ }^{30}$ K. Hämäläinen, M. Krisch, C. Kao, W. Caliebe, and J. B. Hastings, Rev. Sci. Instrum. 66, 1525 (1995).
${ }^{31}$ U. Bergamnn, P. Glatzel, and S. P. Cramer, Microchem. J. 71, 221 (2003).

${ }^{32}$ U. Bergmann, M. Ivanovic, P. Glatzel, and S. P. Cramer, IEEE Trans. Nucl. Sci. 50, 140 (2003).

${ }^{33}$ E. Kleymenov, J. A. van Bokhoven, C. David, P. Glatzel, M. Janousch, R. Alonso-Mori, M. Studer, M. Willimann, A. Bergamaschi, B. Henrich, and M. Nachtegaal, Rev. Sci. Instrum. 82, 065107 (2011).

${ }^{34}$ B.-Y. Shew, R.-S. Huang, D.-J. Wang, S.-Y. Perng, C.-K. Kuan, Y. Q. Cai, P. Chow, M. Schwoerer-Boehning, W. Caliebe, C.-C. Kao, and C. T. Chen, Proc. SPIE 4783, 131 (2002).

${ }^{35}$ R. Verbeni, M. Kocsis, S. Huotari, M. Krisch, G. Monaco, F. Sette, and G. Vanko, J. Phys. Chem. Solids 66, 2299 (2005).

${ }^{36}$ S. Huotari, F. Albergamo, G. Vanko, R. Verbeni, and G. Monaco, Rev. Sci. Instrum. 77, 053102 (2006).

${ }^{37}$ B. Dickinson, G. T. Seidler, Z. W. Webb, J. A. Bradley, K. P. Nagle, S. M. Heald, R. A. Gordon, and I. M. Chou, Rev. Sci. Instrum. 79, 123112 (2008).

${ }^{38}$ B. A. Mattern, G. T. Seidler, M. Haave, J. I. Pacold, R. A. Gordon, J. Planillo, J. Quintana, and B. Rusthoven, Rev. Sci. Instrum. 83, 023901 (2012).

${ }^{39}$ J. Pacold, J. A. Bradley, B. A. Mattern, M. J. Lipp, G. T. Seidler, P. Chow, Y. Xiao, E. Rod, B. Rusthoven, and J. Quintana, J. Synchrotron Radiat. 19, 245 (2012).

${ }^{40}$ J. Hoszowska, J.-Cl. Dousse, J. Kern, and C. Rheme, Nucl. Instrum. Methods Phys. Res. A 376, 129 (1996).

${ }^{41}$ A. Shevelko, Y. Kasyanov, O. Yakushev, and L. Knight, Rev. Sci. Instrum. 73, 3458 (2002).

${ }^{42}$ H. Hayashi, M. Kawata, R. Takeda, Y. Udagawa, Y. Watanabe, T. Takano, S. Nanao, and N. Kawamura, J. Electron Spectrosc. Relat. Phenom. 136, 191 (2004).

${ }^{43}$ J. Hoszowska and J.-Cl. Dousse, J. Electron Spectrosc. Relat. Phenom. 137, 687 (2004).

${ }^{44}$ R. Alonso-Mori, J. Kern, D. Sokaras, T.-C. Weng, D. Nordlund, R. Tran, P. Montanez, J. Delor, V. K. Yachandra, J. Yano, and U. Bergmann, Rev. Sci. Instrum. 83, 073114 (2012).

${ }^{45}$ B. Schmitt, C. Bronnimann, E. F. Eikenberry, F. Gozzo, C. Hormann, R. Horisberger, and B. Patterson, Nucl. Instrum. Methods Phys. Res. A 501, 267 (2003).

${ }^{46}$ See http://www.esrf.eu/computing/scientific/xop2.1/ for software description.

${ }^{47}$ A. Bergamaschi, A. Cervellino, R. Dinapoli, F. Gozzo, B. Henrich, I. Johnson, P. Kraft, A. Mozzanica, B. Schmitt, and X. Shi, J. Synchrotron Radiat. 17, 653 (2010).

${ }^{48}$ E. Kleymenov, J. Sa, J. Abu-Dahrieh, D. Rooney, J. A. van Bokhoven, E. Troussard, J. Szlachetko, O. V. Safonova, and M. Nachtegaal, Catal. Sci. Technol. 2, 373 (2012).

${ }^{49}$ J. Drahokoupil, M. Polcik, and E. Pollert, Solid State Commun. 66, 455 (1988).

${ }^{50}$ D. Zatsepin, V. R. Galakhov, M. A. Korotin, V. V. Fedorenko, E. Z. Kurmaev, S. Bartkowski, M. Neumann, and R. Berger, Phys. Rev. B 57, 4377 (1998) 